\title{
S. Hendrickx: Love, Sex \& Long-Term Relationships: What People with Asperger Syndrome Really Really Want
}

\author{
Jessica Kingsley Publishers, London, UK, 2008, 144 pp, ISBN 978-1-84310-605-0. \\ $\$ 19.95$ (paper)
}

\author{
Amy Drahota
}

Published online: 1 July 2009

(c) The Author(s) 2009. This article is published with open access at Springerlink.com

There is a long history of specialty self-help books regarding love and relationships for adults; however, few books target adults with Asperger Syndrome as the intended audience. Sarah Hendrickx's book, Love, Sex \& LongTerm Relationships: What People with Asperger Syndrome Really Really Want, is a welcome addition to this collection of literature. Written primarily as an instructional guide for adults with Asperger Syndrome (AS) and their partners, this book offers a direct and focused discussion about dating, relationships, love and intimacy, and sexual behaviors. Due to the often limited social interactions that individuals with AS experience throughout their life, Hendrickx attempts to provide the information and lessons that neurotypical individuals often gain through discussions with friends, intimate encounters with partners, and subtle social cues in a straightforward and concrete manner. The information and perspectives presented in this book are derived from Hendrickx's own experience-she is a neurotypical partner of a man with AS-and also from surveys and interviews from single individuals with AS, those in relationships, and their partners-some with AS and others without.

Each of the 11 chapters introduce a topic related to relationships, love, and sex with a brief discussion of the topic and specific difficulties that may be experienced by individuals with AS. For example, Chap. 3: Love and

\footnotetext{
A. Drahota $(\bowtie)$

Department of Psychiatry, University of California,

San Diego, San Diego, CA, USA

e-mail: adrahota@ucsd.edu

\section{A. Drahota}

Child and Adolescent Services Research Center,

Rady Children's Hospital, 3020 Children's Way

(MC 5033), San Diego, CA 92123, USA
}

Intimacy begins by discussing the desire of many for "emotional closeness and intimacy" (p. 31) in their relationships then transitions to a discussion regarding difficulties that many individuals with AS have "understanding and responding to emotional requirements of a partner" (p. 31). Exemplifying these difficulties, Hendrickx includes statements by individuals who responded to her surveys and interviews. For example a neurotypical female responded, "I asked my husband (AS) to please be nice to me. He looked at me blankly and said: I have absolutely no idea how to be nice to you." (p. 38). The comments included in each chapter wonderfully elucidate the varied opinions, attitudes, and experiences of individuals with AS and their partners.

From the introduction and the comments provided by individuals with AS and their partners, Hendrickx provides a basic guide for individuals with AS. This guidance includes the rationale for specific actions and attitudes of neurotypical individuals, strategies to overcome difficulties resulting from AS symptoms, and a common language to assist with discussing relationship issues between partners. The guidance provided is open-minded and supportive to individual preferences, and promotes respect, consent, and emotional and physical health (e.g., "For the person with AS, asking for guidance on how to help and support a partner may be greatly appreciated and is better than doing nothing at all or waiting for a request to be made" (p. 48)). Further, the guidance relates to the multiple facets of human sexuality and relationships. Included in each chapter are subtopics that seem associated with the more general chapter heading. This organizational style, along with an index in the back of the book, may perhaps assist individuals with AS and their partners to understand the multidimensionality of each topic and provide guidance ranging from general to specific. Further, she concludes her 
book with general findings and impressions gathered from her surveys and interviews.

One limitation of this book, is the lack of reference to empirical (peer reviewed) literature supporting her guidance and instruction to adults with AS. Instead, she draws heavily from popular press books and what may be considered as "common sense". The popular press books range from those written by researchers (e.g., Attwood 2006; Baron-Cohen 2003) to those written by individuals with AS or living with partners with AS that use similar methodology (e.g., personal perspectives, surveys, interviews with others) (e.g., Stanford 2003; Lawson 2005). Currently, there is a paucity of literature regarding sexuality that involves adults with AS (see Sullivan and Caterino 2008). While a literature base is emerging, very few studies focus on the knowledge, attitudes, interests, and experience of adults with AS (Konstantareas and Lunsky 1997; Ousley and Mesibov 1991). Additionally, Hendrickx may value individual accounts to the AS experience above and beyond what the empirical literature can provide. On the other hand, there is a great deal of empirical literature regarding these topics involving neurotypical individuals which may have been drawn upon to support the guidance and instruction that she provides. Of course, it is unclear how the attitudes and experiences of individuals with AS differ from the neurotypical population and this may be the reason for her limiting the references specifically to written works involving individuals with AS.

A valuable feature of this book is the inclusion of verbatim comments provided by the respondents to her survey and interview questions. Readers are able to get intimate details about others' experiences and attitudes regarding dating, relationships, sexual activities and preferences, and infidelity. The depth and breadth of these responses may provide great comfort to individuals with AS who might not have opportunities to compare their activities and attitudes to others. Further, individuals with AS may be able to analyze the responses by neurotypical partners to better understand their actions and emotions, and enhance their own social relationships. In addition, neurotypical partners of individuals with AS may learn about and understand the difficulties that individuals with AS face in dynamic social interactions and intimate relationships. Interestingly, there were a range of responses by neurotypical partners. Some neurotypical individuals seemed to recognize and accept the difficulties experienced by their partners with AS and appreciated the effort and displays of affection, for example, that their partners are able to show.
Others reported significant challenges in their relationships as a result of the symptoms displayed by their partner with AS and indicated an overall dissatisfaction with the relationship. The comments made by these partners may especially hit home for readers who are the neurotypical partner in their own relationship and are attempting to better understand their partner with AS. Fortunately, as stated by Hendrickx, evidence abounds throughout this book that "mutually satisfying personal and sexual relationships are happily occurring with the Asperger Syndrome (AS) population" (p. 137).

Overall, this book is recommended for adults with AS and their partners. It provides a concise yet fairly comprehensive set of topics regarding relationships, love and sex and offers a common language for individuals to begin thinking and communicating about with their partners. By highlighting the unique characteristics of individuals with AS, it emphasizes the possible difficulties that individuals with AS may experience in social interactions and relationships. Finally, this book offers clear strategies for overcoming potential barriers to successful relationships that can be utilized by motivated individuals with AS and their partners.

Open Access This article is distributed under the terms of the Creative Commons Attribution Noncommercial License which permits any noncommercial use, distribution, and reproduction in any medium, provided the original author(s) and source are credited.

\section{References}

Attwood, T. (2006). The complete guide to Asperger Syndrome. London, UK: Jessica Kingsley.

Baron-Cohen, S. (2003). The essential difference: Men, women and the extreme male brain. London, UK: Penguin.

Konstantareas, M. M., \& Lunsky, Y. J. (1997). Sociosexual knowledge, experience, attitudes, and interests of individuals with autistic disorder and developmental delay. Journal of Autism and Developmental Disorders, 27, 397-413.

Lawson, W. (2005). Sex, sexuality and the autism spectrum. London, UK: Jessica Kingsley.

Ousley, O. Y., \& Mesibov, G. B. (1991). Sexual attitudes and knowledge of high-functioning adolescents and adults with autism. Journal of Autism and Developmental Disorders, 21, 471-481.

Stanford, A. (2003). Asperger Syndrome and long-term relationships. London, UK: Jessica Kingsley.

Sullivan, A., \& Caterino, L. C. (2008). Addressing the sexuality and sex education of individuals with autism spectrum disorders. Education and Treatment of Children, 31, 381-394. 